Publ. RIMS, Kyoto Univ.

25 (1989), 1-19

\title{
Global Moduli Spaces and Simple Holomorphic Bundles
}

\author{
By \\ Siegmund Kosarew* and Christian OKONEK*
}

The original motivation for writing this paper was to give a proof for the existence of the moduli space of simple holomorphic bundles $\mathcal{E}$ (i. e. $\operatorname{End}(\mathcal{E})=\boldsymbol{C}$ ) on a fixed compact complex space $X$. This is achieved in (6.5). The method, used by us, is so general that we can formulate an abstract sufficient criterion for the representability of analytic functors (see theorem (2.2)). Even simple coherent sheaves can be treated in this way (theorem (6.4)). Some special cases were obtained formerly by A. Norton in $[\mathbf{N o}]$, where $X$ has to be a manifold. A more explicit construction of the moduli space of simple bundles in the kählerian case is given in [Lü-Ok] by a completely different approach.

Our representability theorem (2.2) is in some sense more special than the one's known in the literature (for example see $[\mathbf{B i}]_{2}$ or $[\mathbf{S}-\mathbf{V}]$ ), but has the advantage to fit into the philosophy of constructing moduli spaces as quotients with respect to group operations. It is also necessary here to consider relative group operations on complex spaces and to construct relative quotients. The material that we need, is contained in $\S 3$.

We also deal with the existence of coarse moduli spaces of analytic functors in general. This notion is weaker than that of the representability of the associated sheafified functor. The main result is formulated in theorem (2.3). It should be mentioned that this theorem is inspired by the work of G. Schumacher, see $[\mathbf{S c h u}]_{1},[\mathrm{Schu}]_{2}$.

This paper grew out from discussions of both authors at the Sonderforschungsbereich 170 "Geometrie und Analysis" in Göttingen. We would like to express our sincere thanks to the SFB, especially for the stimulating atmosphere. Last not least, H. Flenner was very interested in our work and we want to thank him for several useful remarks.

Communicated by M. Kashiwara, March 31, 1987.

* Sonderforschungsbereich 170, "Geometrie und Analysis" Math. Inst. d. Universitat, Bunsenstrasse 3/5, D-3400 Göttingen, Federal Republic of Germany. 


\section{$\S 1$. Notations}

(1.1) In the following we denote by (An) resp. (Gan) the category of complex spaces resp. complex space germs. For a complex space $S$ resp. a complex space germ $S=(S, 0)$, we define $(\mathrm{An} / S)$ resp. (Gan/S) to be the category of complex spaces over $S$ resp. of complex space germs over $(S, 0)$.

Let $S$ be a complex space. A groupoid over $(\mathrm{An} / S)$ is a category $F$ together with a covariant functor

$$
p: F \longrightarrow(\mathrm{An} / S)
$$

such that each fibre category $F(T)$, where $T$ is a space of $(\mathrm{An} / S)$, is a groupoid in the usual sense (i.e. the objects of $F(T)$ form a set and each morphism in $F(T)$ is an isomorphism) and, moreover, for each object $a$ of $F(T)$ and $S$-morphism $T^{\prime} \rightarrow T$ the "base-change" $a_{T^{\prime}}$ of $a$ with respect to $T^{\prime} \rightarrow T$ is defined in a unique manner (for a precise definition see for instance $[\mathbf{B i}]_{1} \S 1$ ). By passing to the isomorphy classes, a groupoid gives a contravariant functor

$$
\begin{gathered}
|F|:(\mathrm{An} / S) \longrightarrow \text { (sets) } \\
T \longmapsto|F(T)| .
\end{gathered}
$$

Vice versa, any contravariant functor $(\mathrm{An} / S) \rightarrow$ (sets) induces in an obvious way a groupoid over $(\mathrm{An} / S)$. Observe that these two constructions are not inverse to each other.

(1.2) Let $F:(\mathrm{An} / S) \rightarrow($ sets $)$ be a contravariant functor. Then $F$ is called representable (i.e. $F$ possesses a fine moduli space), if there exists a complex space $X$ in $(\mathrm{An} / S)$ together with an isomorphism $F \rightarrow h_{X / S}$ of functors (here $h_{X / S}$ denotes the functor $\operatorname{Hom}_{S}(-, X)$ ). We say that $F$ has a coarse moduli space, if the covariant functor

$$
\begin{gathered}
(\mathrm{An} / S) \longrightarrow(\text { sets }) \\
X \longmapsto \operatorname{Hom}_{S}\left(F, h_{X / S}\right)
\end{gathered}
$$

is representable by a complex space $Z$ over $S$, such that the canonical mapping

$$
\left.F(\operatorname{Spec}(\kappa(s))) \longrightarrow\left|Z_{s}\right| *\right)
$$

is bijective for each point $s \in S$. Obviously a fine moduli space is also a coarse one.

(1.3) Let $F \rightarrow(\mathrm{An} / S)$ be a groupoid, $S^{\prime}$ a space in $(\mathrm{An} / S)$ and $a, b$ objects of $F\left(S^{\prime}\right)$. Then we can define the following two contravariant functors

*) Here $\kappa(s)=\mathcal{O}_{S, s / m_{s}} \cong C$ denotes the local field in $s \in S$. 


$$
\begin{aligned}
\operatorname{Isom}_{S^{\prime}}^{F}(a, b):\left(\mathrm{An} / S^{\prime}\right) \longrightarrow(\text { sets }) & \\
T & \longmapsto \operatorname{Isom}_{T}\left(a_{T}, b_{T}\right), \\
N_{S^{\prime}}^{F}(a, b):\left(\operatorname{An} / S^{\prime}\right) \longrightarrow(\text { sets }) & \\
T & \longmapsto \begin{cases}\varnothing, & \text { if } \operatorname{Isom}_{T}\left(a_{T}, b_{T}\right)=\varnothing \\
\{\varnothing\}, & \text { otherwise. }\end{cases}
\end{aligned}
$$

and get a natural $S^{\prime}$-morphism

$$
\operatorname{Isom}_{S^{\prime}}^{F}(a, b) \longrightarrow N_{S^{\prime}}^{F}(a, b) .
$$

In the case $a=b$, we put

$$
A u t_{S^{\prime}}^{F}(a):=\operatorname{Isom}_{S^{\prime}}^{F}(a, a) .
$$

Obviously, this last functor has even values in the category of groups. Furthermore, $\operatorname{Isom}_{S^{\prime}}^{F}(a, b)$ is always a formal principal homogeneous space under the left action of $A u t_{S^{\prime}}^{F}(a)$.

(1.4) Remark. In the situation of (1.3), one has a bijection

$$
\operatorname{Image}\left(\operatorname{Isom}_{S^{\prime}}^{F}(a, b) \stackrel{\alpha}{\longrightarrow} h_{S^{\prime} / S^{\prime}}\right) \stackrel{\sim}{\longrightarrow} N_{S^{\prime}}^{F}(a, b),
$$

where $\alpha$ denotes the "structure" functor.

Let $a=b$ and $p r_{i}: S^{\prime} \times{ }_{S} S^{\prime} \rightarrow S^{\prime}$ be the canonical projection for $i=1,2$. Then the double arrow

$$
\operatorname{Isom}_{S^{\prime} \times S^{\prime}}^{F}\left(p r_{1}^{*}(a), p r_{2}^{*}(a)\right) \Longrightarrow h_{S^{\prime} / S}
$$

defines a pre-equivalence relation*) on $h_{S^{\prime} / S}$.

(1.5) Let $F:(\mathrm{An} / S) \rightarrow$ (sets) be a contravariant functor. We say that $F$ is a sheaf or of local nature, if for each complex space $X$ in $(\mathrm{An} / S)$, the restriction of $F$ to the open subsets of $X$ is a sheaf. As usual, to an arbitrary $F$ one can construct its "sheafification" $F$ " and one has a canonical morphism $F \rightarrow F^{\#}$ of functors with the obvious universal property.

(1.6) Remark. If $F^{\#}$ is representable, then $F$ has a coarse moduli space.

Proof. Assume that $F^{\#} \cong h_{Z / S}$ with a complex space $Z$ in $(\mathrm{An} / S)$. Then, for each $X$ over $S$ one has

$$
\begin{aligned}
\operatorname{Hom}_{S}\left(F, h_{X / S}\right) & =\operatorname{Hom}_{S}\left(F^{\#}, h_{X / S}\right) \\
& =\operatorname{Hom}_{S}\left(h_{Z / S}, h_{X / S}\right) \\
& =\operatorname{Hom}_{S}(Z, X)
\end{aligned}
$$

*) the notion of a pre-equivalence relation is taken here from [Ki]. 
and $F(\operatorname{Spec}(\kappa(s)))=F^{\#}(\operatorname{Spec}(\kappa(s)))=\left|Z_{s}\right|$ for $s \in S .-$

(1.7) Let $p: F \rightarrow(\mathrm{An} / S)$ be a groupoid, $S^{\prime}$ a space in $(\mathrm{An} / S)$ and $a$ an object of $F\left(S^{\prime}\right)$. For each $S$-morphism $\alpha: S^{\prime} \rightarrow T$, the fibre category

$$
F_{a}(T)
$$

is defined in the following way: The objects of $F_{a}(T)$ are morphisms $\varphi_{b}: a \rightarrow b$, where $b$ is an object of $F(T)$, with $p\left(\varphi_{b}\right)=\alpha$. A morphism $\psi: b \rightarrow c$ in $F_{a}(T)$ is a morphism in $F(T)$, such that $\phi \varphi_{b}=\varphi_{c}$ and $p(\psi)=i d_{T}$.

For the standard notions of deformation theory, like versal, seni-universal, formal versal etc., we refer to the conventions used in $[\mathbf{B i}]_{1}$.

\section{§2. Suficient Criteria for Representability and for the Existence of Coarse Moduli Spaces}

We are going now to formulate some assertions which give sufficient conditions for representability or the existence of coarse moduli spaces. For this purpose, we shall use the following criterion of Schuster/Vogt from [S-V] Prop. (1.1), which will be stated in the relative context.

(2.1) Theorem. Let $F:(\mathrm{An} / S) \rightarrow($ sets $)$ be a contravariant functor with the following properties

(1) $F$ is of local nature,

(2) for each point $s \in S$ and $a_{0} \in F(\operatorname{Spec}(\kappa(s)))$, there exists a gerin $\left(S^{\prime}, s^{\prime}\right)$ over $(S, s)$ and an element $a \in F\left(S^{\prime}\right)$ with $a_{s^{\prime}}=a_{0}$, sucin that $a$ is a formal versal deformation of each $a_{x}$ for $x \in S^{\prime}$,

(3) if $S^{\prime}$ is a space in $(\mathrm{An} / S)$ and $a, b \in F\left(S^{\prime}\right)$ are two elements, then the functor (see (1.3))

$$
N_{S^{\prime}}^{F^{\prime}}(a, b):\left(\mathrm{An} / S^{\prime}\right) \longrightarrow(\text { sets })
$$

is representable by a locally closed (resp. closed) analytic subspace of $S^{\prime}$.

Then $F$ is representable (resp. representable by a separated morphism).

We are going to apply this criterion to the following situation.

(2.2) Theorem. Let $F \rightarrow(\mathrm{An} / S)$ be a groupoid and $|F|$ the associated isomorphy class functor. Assume that

(1) $|F|$ satisfies condition (2.1) (2),

(2) if $S^{\prime}$ is a space in $(\mathrm{An} / S)$ and $a, b$ are objects of $F\left(S^{\prime}\right)$, then

(i) the functor $I_{\operatorname{som}_{S^{\prime}}}^{F}(a, b)$ is representable by a separated morphism $Z(a, b) \rightarrow S^{\prime}$,

(ii) the Lie group $G(a):=Z(a, a)$ is smooth over $S^{\prime}$,

(iii) the operation of $G(a)$ on $Z(a, b)$ is always locally proper (resp. proper) in the sense of (3.12) (2). 
Then the functor $|F| \#$ is representable (resp. representable by a separated morphism). Especially $|F|$ has a coarse moduli space.

In $\S 3$ we are going to discuss condition (2.2) (iii). Next, we state a theorem concerning the existence of coarse moduli spaces.

(2.3) Theorem. Let $F \rightarrow(\mathrm{An} / S)$ be a groupoid. With the notations of (2.2), assume that

(1) for each $s \in S$ and $a_{0} \in F(\operatorname{Spec}(\kappa(s)))$, the local groupoid $F_{a_{0}} \rightarrow(\operatorname{Gan} /(S, s))$ satisfies the conditions $(\mathbf{S} 1)^{\prime},(\mathbf{S} 2)$, (S3) from $[\mathbf{B i}]_{1}$ and that there are convergent formal semi-universal deformations; moreover, openness of formal versality holds,

(2) if $S^{\prime}$ is a space in $(\mathrm{An} / S)$ and $a, b$ are objects of $F\left(S^{\prime}\right)$, then

(i) the functor $\operatorname{Isom}_{S^{\prime}}^{F}(a, b)$ is representable by a separated morphism $Z(a, b) \rightarrow S^{\prime}$,

(ii) the structure map $G(a) \rightarrow S^{\prime}$ is smooth along the unit section,

(iii) if $G(a)^{0}$ denotes the relative component of the unit section (see (3.4)), the morphism $Z(a, b) / G(a)^{0} \rightarrow S^{\prime}$ is locally finite*) (resp. finite).

Then $|F|$ has a coarse moduli space (resp. one with a separated structure map).

\section{$\S 3$. Relative Complex Lie Groups and Operations}

In this section we consider operations of (relative) complex Lie groups which are important for the construction of global moduli spaces.

(3.1) Definition. A complex Lie group over $S$ is by definition a mapping $\pi:{ }^{\prime \prime} G \rightarrow S$ of complex spaces together with $S$-morphism

$$
\begin{array}{ll}
\varepsilon: S \longrightarrow G & \text { (unit section), } \\
m: G \times{ }_{S} G \longrightarrow G & \text { (multiplication), } \\
j: G \longrightarrow G & \text { (inversion) }
\end{array}
$$

such that the usual properties for a group object in $(\mathrm{An} / S)$ hold.

(3.2) Remark. The mapping $\pi: G \rightarrow S$ is separated (i.e. the diagonal map $\Delta_{G / S}: G \rightarrow G \times{ }_{S} G$ is a closed embedding), iff the unit section $\varepsilon: S \rightarrow G$ is a closed embedding (compare also [SGA] Exp. $V I_{B}$, Prop. 5.1).

In the following the word "Lie group" always means separated complex Lie group (over $S$ ).

(3.3) Lemma. Let $\pi: G \rightarrow S$ be a Lie group and $V \subset G$ an open subset, such that $\pi \mid V: V \rightarrow S$ is smooth. Then the mappings $m: V \times{ }_{S} G \rightarrow G$ and $m: G \times{ }_{S} V \rightarrow G$ *) for this notion see (3.12) (1). 
are smooth too.

Proof. As $\left(i d_{v}, m\right): V \times{ }_{S} G \rightarrow V \times{ }_{S} G$ is an isomorphism, it is sufficient to show that the canonical projection $p r_{2}: V \times_{S} G \rightarrow G$ is smooth. But this is clear, since $V \rightarrow S$ is smooth. The case $m: G \times{ }_{S} V \rightarrow G$ is done analogously.-

The following assertion gives a criterion for the existence of the relative 1-component of a Lie group.

(3.4) Proposition. Let $\pi: G \rightarrow S$ be a Lie group over $S$, such that $\pi$ is smooth in a neighbourhood of $\varepsilon(S)$. Then there exists an open Lie subgroup $G^{\circ}$ of $G$ with the following properties:

(1) The fibres $\left(G^{\circ}\right)_{s}$ are connected for $s \in S$ and $\left|G^{\circ}\right|=\bigcup_{s \in S}\left|\left(G_{s}\right)^{\circ}\right|$.

(2) $G^{\circ} \rightarrow S$ is smooth.

(3) For each space $T$ over $S$ one has $\left(G_{T}\right)^{\circ}=\left(G^{\circ}\right)_{T}$.

Proof. We fix at first an open neighbourhood $W$ of $\varepsilon(S)$ in $G$ with the properties

(i) all fibres $W_{s}, s \in S$, are connected,

(ii) $\pi: W \rightarrow S$ is smooth.

Putting $V:=W \cup j(W)$, we see that $V$ also satisfies (i) and (ii)。 Inductively, we define $V^{k} \subset G$ to be

$$
\begin{aligned}
& V^{1}:=V, \\
& V^{k+1}:=m\left(V \times_{S} V^{k}\right) \quad \text { for } \quad k \geqq 1 .
\end{aligned}
$$

According to the lemma, each $V^{k}$ is open in $G$ and, moreover, fulfills (i) and (ii). Now we set

$$
G^{\circ}:=\bigcup_{k \in N} V^{k} \text {. }
$$

By construction, $G^{\circ}$ is invariant under $m$ and $j$ and contains $\varepsilon(S)$. Obviously, (i) and (ii) hold for $G^{\circ}$ and $\left(G^{\circ}\right)_{s}=\left(G_{s}\right)^{\circ}$ for $s \in S$. This shows (1) and (2).

Fix a space $T$ in $(\mathrm{An} / S)$. The canonical map $\left(G_{T}\right)^{\circ} \rightarrow G$ factors through $G^{\circ}$ 。 Therefore also (3) holds.-

(3.5) Remark. The construction of $G^{\circ}$ is compatible with Lie group homomorphisms, i. e. if $f: G \rightarrow H$ is a homomorphism of Lie groups over $S$, then $f$ induces a homomorphism $f^{\circ}: G^{\circ} \rightarrow H^{\circ}$.

(3.6) Notation. Let $G \rightarrow S$ be a Lie group over $S$ which is smooth over $S$ along $\varepsilon(S)$. Then $G$ is called (globally) decomposed, if one has

$$
G=\underset{\nu \in I}{\Perp} G^{\nu}
$$


with $o \in I$ and open subsets $G^{\nu} \subset G$, which map surjectively on $S$ and are fibrewise connected.

(3.7) Definition. Let $G \rightarrow S$ be a Lie group over $S$ and $\omega: G \times{ }_{S} Z \rightarrow Z$ a relative operation of $G$ on the complex space $Z$ over $S$. Then $Z$ is called a formal principal homogeneous space (with resp. to $G$ ), if the map

$$
\left(p r_{2}, \omega\right): G \times{ }_{S} Z \rightarrow Z \times{ }_{S} Z
$$

is an isomorphism.

(3.8) Lemma. Let $G$ be a decomposed Lie group, smooth over $S$, and $Z$ a formal principal homogeneous space. Then for each connected component $Z^{\nu}$ of $Z$, there exists an open Lie subgroup $H^{\nu}$ of $G$, such that

$$
\left(p r_{2}, \omega\right): H^{\nu} \times{ }_{s} Z^{\nu} \longrightarrow Z^{\nu} \times{ }_{s} Z^{\nu}
$$

is a well-defined isomorphism.

Proof. We may assume $S$ to be connected. Let $G=\Perp_{\mu} G^{\mu}$ be the decomposition of $G$ according to (3.6) and $Z=\Perp_{2} Z^{\nu}$ the partition into connected components. Now

$$
\underset{\mu, \nu}{\Perp}\left(G^{\mu} \times{ }_{S} Z^{\nu}\right) \stackrel{\left(p r_{2}, \omega\right)}{\longrightarrow} \underset{\nu, \nu^{\prime}}{\Perp}\left(Z^{\nu} \times{ }_{S} Z^{\nu^{\prime}}\right)
$$

is an isomorphism. As $G^{\mu} \rightarrow S$ is surjective and smooth with connected fibres, one sees that $G^{\mu} \times{ }_{S} Z^{\nu}$ is connected. Therefore there is a union $H^{\nu}$ of certain $G^{\mu}$ (with $H^{\nu} \supset G^{\circ}$ ), such that

$$
H^{\nu} \times{ }_{S} Z^{\nu} \longrightarrow Z^{\nu} \times{ }_{S} Z^{\nu}
$$

is an isomorphism. We must show now that $H^{\nu}$ is invariant under $m_{G}$ and $j_{G}$. Let $G^{\mu}, G^{\mu^{\prime}}$ be components of $H^{\nu}$ and $G^{\mu \mu^{\prime}}$ the image of $m_{G}: G^{\mu} \times{ }_{S} G^{\mu^{\prime}} \rightarrow G$ which is open and connected. The commutative diagram

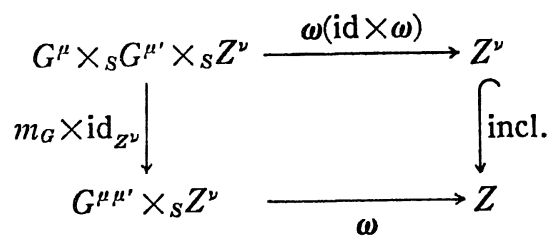

gives $G^{\mu \mu^{\prime}} \subset H^{\nu}$. The invariance of $H^{\nu}$ with respect to $j_{G}$ is immediate.-

(3.9) Notation. In the situation of lemma (3.8), the Lie group $H^{\nu}$ is called the Lie subgroup of $G$ belonging to the component $Z^{\nu}$.

Now, we shall show an important assertion, derived from the main theorem 
of $[\mathrm{Ki}]$.

(3.10) Proposition. Let $G \rightarrow S$ be a Lie group, smooth over $S$, and $\omega: G \times{ }_{S} Z \rightarrow Z$ an operation, such that $\left(p r_{2}, \omega\right): G \times{ }_{S} Z \rightarrow Z \times{ }_{s} Z$ is an open embedding. Then

(1) the quotient $Z / G$ exists in the category of complex spaces and is a space over $S$ in a natural way,

(2) the quotient map $Z \rightarrow Z / G$ is smooth,

(3) the canonical map $G \times{ }_{S} Z \rightarrow Z \times{ }_{Z I G} Z$ is an isomorphism,

(4) the construction of $Z / G$ commutes with base change, i.e. $(Z / G)_{T}=Z_{T} / G_{T}$ for each space $T$ over $S$,

(5) if we denote the associated functor to $Z$ resp. $G$ on $(\mathrm{An} / S)$ by $\tilde{Z}$ resp. $\tilde{G}$, the associated sheaf of $\tilde{Z} / \tilde{G}$ identifies with $(Z / G)^{2}$.

As an immediate consequence, we note

(3.11) Remark. Let $G \rightarrow S$ be a Lie group, smooth along $\varepsilon(S)$ over $S$. Then the quotient $G / G^{\circ}$ exists and is a discrete Lie group over $S$ in a natural way. If $G / G^{\circ} \rightarrow S$ is an unramified covering, then $G \rightarrow S$ is, locally in $S$, decomposed.

Proof of (3.10). Properties (1) and (3) follow immediately from Kiehl's theorem ([Ki] Theorem (2.1)) and also the faithful flatness of $Z \rightarrow Z / G$. The smoothness of this map is seen by using (3) and the fact that $G \rightarrow S$ is smooth. For the verification of (4) we must show that the canonical $T$-morphism $Z_{T} / G_{T} \rightarrow(Z / G)_{T}$ is an isomorphism. We put $Y:=Z / G$ for abbreviation. Now $Z \rightarrow Y$ is a universal effective epimorphism and therefore we have the two exact sequences

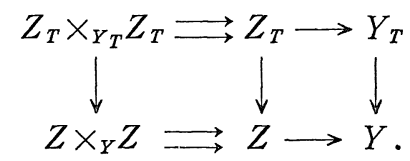

Since $\left(G \times{ }_{S} Z\right)_{T}=Z_{T} \times_{Y_{T}} Z_{T}$ (compare (3)), we get $Y_{T}=Z_{T} / G_{T}$. Property (5) follows from the bijectivity of the natural map $(\tilde{Z} / \tilde{G})^{\#} \rightarrow(Z / G)^{\sim}$ which is a consequence of (2) and (3).-

(3.12) Definition. (1) Let $f: X \rightarrow S$ be a continuous map of topological spaces. Then $f$ is called locally finite, iff for each point $s \in f(X)$ there is an open neighbourhood $S^{\prime}$ of $s$ in $S$, such that $f \mid S^{\prime}: f^{-1}\left(S^{\prime}\right) \rightarrow S^{\prime}$ is finite in the usual sense.

(2) Let $G \rightarrow S$ be a Lie group and $\omega: G \times{ }_{S} Z \rightarrow Z$ an operation, such that for each open subset $U \subset S$ the quotient $Z_{U} / G_{U}$ exists as a complex space and identifies with $(Z / G)_{U}$. Then we call $\omega$ locally proper (resp. proper) as an operation, iff for each point $s \in \operatorname{Im}(Z \rightarrow S)$ (resp. $s \in S$ ), there exists an open neighbourhood $U$ of $s$ in $S$ and a subset $A \subset Z_{U}$, such that the composition $A \rightarrow Z_{U} \rightarrow U$ is proper and $A \rightarrow(Z / G)_{U}$ is surjective. 
(3.13) Proposition. In the situation of (3.10), let $\omega$ be locally proper (resp. proper) and $Z$ a formal principal homogeneous space. Then $Z / G \rightarrow S$ induces an isomorphism onto a locally closed (resp. closed) analytic subspace $S^{\prime}$ of $S$.

Proof. By (3.10) (4), the map $Z / G \rightarrow S$ is injective and around each point $\bar{z} \in Z / G$ a closed embedding of complex space germs. The assertion follows now from (3.12) (2).-

(3.14) Definition. Let $f: Z \rightarrow S$ be a morphism of complex spaces. Then $f$ is called of locally constant connectedness type, iff the following two properties are fulfilled.

(1) For each point $s \in S$, there is a fundamental system $\left(U_{\nu}\right)_{\nu \in N}$ of open connected neighbourhoods with $U_{\nu} \supset U_{\nu+1}$ and such that the decomposition of $Z_{U_{\nu}}$ into connected components commutes with restriction to $U_{2+1}$ and there are only finitely many such components.

(2) Every fibre $Z_{s}$ has a finite number of connected components and each component of $Z_{U_{\nu}}$ meets $Z_{s}$, in the case $Z_{s} \neq \varnothing$.

(3.15) Remark. Assume that $f: Z \rightarrow S$ is relatively compactifiable, i.e. there is a proper holomorphic mapping $\bar{Z} \rightarrow S$ and a Zariski-open $S$-embedding $Z \rightarrow \bar{Z}$. Then $f$ satisfies condition (3.14) (1) (but not (3.14)(2) in general). This is a consequence of $[\mathbf{B i}-\mathbf{F l}]$ Theorem (4.2) together with [Go-MP], proof of Theorem on p. 84, applied to the constructible sheaf $f_{*}\left(\boldsymbol{Z}_{Z}\right)$.

(3.16) Theorem. Let $G \rightarrow S$ be a Lie group and $\omega: G \times{ }_{s} Z \rightarrow Z$ an operation. Assume that

(1) $G \rightarrow S$ is smooth and, locally in $S$, decomposed,

(2) $Z$ is formal principal homogeneous,

(3) $Z \rightarrow S$ is of locally constant connectedness type.

Then $\omega$ is locally proper as an operation. Especially $Z / G \rightarrow S$ is an isomorphism onto a locally closed complex subspace of $S$.

Proof. Let $z \in Z$ be a point with image $s$ in $S$. After shrinking $S$ around $s$, we may assume that $Z$ is a union $Z=Z_{1} \sqcup \cdots\left\llcorner Z_{k}\right.$ of finitely many connected components and that this decomposition is compatible with restriction to a fundamental system $\left(U_{\imath}\right)_{i}$ of neighbourhoods of $s$ like in (3.14). Let $G_{\nu}$ be the component belonging to $Z_{\text {ע }}$ (compare (3.9)), so

$$
\left(p r_{2}, \omega\right): G_{\nu} \times{ }_{S} Z_{\nu} \longrightarrow Z_{\nu} \times{ }_{S} Z_{\nu}
$$

is an isomorphism. Assume that $z \in Z_{\nu}$ for some $\nu \in\{1, \cdots, k\}$. By the existence of relative transversal slices (see $[\mathbf{K o}](\mathbf{3 . 5})$ ), there is a subgerm $\left(S_{\nu}, z\right)$ of $\left(Z_{\nu}, z\right)$, such that 


$$
\left(G_{\nu}, 1_{s}\right) \times_{(S, s)}\left(S_{\nu}, z\right) \stackrel{\omega}{\longrightarrow}\left(Z_{\nu}, z\right)
$$

is an isomorphism and, moreover, the composition $\left(S_{\nu}, z\right) \rightarrow\left(Z_{\nu}, z\right) \rightarrow(S, s)$ is an embedding. After replacing $S$ by a sufficiently small $U_{\imath}$, we get (by using the smoothness of $G_{\nu} \rightarrow S$ ) that

$$
\omega: G_{\nu} \times{ }_{S} S_{\nu} \longrightarrow Z_{\nu}
$$

is an open embedding. On the other hand, by $\left(^{*}\right)$

$$
G_{\nu} \times{ }_{S} S_{\nu} \longrightarrow S_{\nu} \times{ }_{S} Z_{\nu}=\left(Z_{\nu}\right)_{S_{\nu}}
$$

is an isomorphism. Therefore $\left(Z_{\nu}\right)_{S_{\nu}}$ is open and closed in $Z_{\nu}$, i. e. $\left(Z_{\nu}\right)_{S_{\nu}}=Z_{\nu}$. Summarizing, we see that $S_{\nu} \rightarrow Z_{\nu} / G_{\nu}$ is surjective and $S_{\nu} \rightarrow S$ is a closed embedding. This construction can be done for each $Z_{\nu}$, since $Z_{\nu} \cap Z_{s} \neq \varnothing$ according to (3.14) (2). Now we put

$$
A:=\prod_{\nu=1}^{k} S_{\nu} .
$$

Then the map $A \rightarrow Z / G$ is obviously surjective and $A \rightarrow S$ is proper. This gives the theorem.-

At the end of this section, we just want to mention the following trivial but important lemma.

(3.17) Lemma. Let $G$ be a finite group, operating on a complex space germ $(X, 0)$. Then there is a fundamental system of invariant open neighbourhoods of 0 in $X$.

\section{$\S 4$. Proof of Theorem $(2.2)$}

We want to apply the general criterion $(\mathbf{2 . 1})$ to the sheafified functor $|F|^{\#}:(\mathrm{An} / S) \rightarrow($ sets $)$ associated to $|F|$. By construction, $|F|^{\#}$ is of local nature. If $|F|$ fulfills (2.1) (2), then so does $|F|^{\#}$.

Now, let $S^{\prime}$ be a space in $(\mathrm{An} / S)$ and $a^{\prime}, b^{\prime}$ two elements in $|F|^{\#}\left(S^{\prime}\right)$. We have to show that the functor

$$
N_{S^{\prime}}^{\prime F^{\prime} \#}\left(a^{\prime}, b^{\prime}\right):\left(\mathrm{An} / S^{\prime}\right) \longrightarrow \text { (sets) }
$$

(see (1.3)) is representable by a locally closed (resp. closed) complex subspace of $S^{\prime}$. We immediately reduce the situation to the case where $a^{\prime}$ and $b^{\prime}$ are induced by objects $a$ and $b$ in $F\left(S^{\prime}\right)$, otherwise use a sufficiently fine open covering of $S^{\prime}$. We set $Z:=Z(a, b)$ and $G:=G(a)$. Then we get a morphism of functors

$$
\tilde{Z} \longrightarrow N_{S^{\prime}}^{\mid F^{\prime} \#}\left(a^{\prime}, b^{\prime}\right)
$$


which induces an isomorphism

$$
(\tilde{Z} / \tilde{G})^{\#} \longrightarrow N_{S^{\prime}}^{\prime F^{\prime}}\left(a^{\prime}, b^{\prime}\right) .
$$

Here $\tilde{Z}$ and $\tilde{G}$ are the associated functors to $Z$ and $G$ on $(\mathrm{An} / S)$ and "\#” means sheafification. The assertion follows now from (3.13) together with (3.10) (5) and the assumptions made in (2.2) (2).-

\section{§5. Proof of Theorem $(2.3)$}

First we note that, using $[\mathbf{B i}]_{1}(\mathbf{3 . 2})$, it is possible to replace in (2.3) (1) the word "formal" by "convergent".

The construction of the "local pieces" of the coarse moduli space goes as follows : Fix a point $s \in S$ and an object $a_{0}$ in $F(\operatorname{Spec}(\kappa(s)))$. According to (2.3) (1), there is a complex space $S^{\prime}$ over $S$, a point $s^{\prime} \in S^{\prime}$, which maps to $s$, and an object $a$ in $F\left(S^{\prime}\right)$ with $a_{s^{\prime}} \cong a_{0}$, such that $a$ is versal deformation of each $a_{t}$ for $t \in S^{\prime}$. We show now that $a$ is even universal in $s^{\prime}$. For this purpose we consider a deformation situation

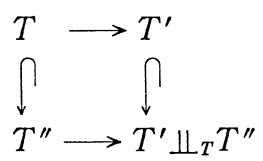

(see $\left.[\mathbf{B i}]_{1}(\mathbf{1 . 2})\right)$ of artinian germs over $(S, s)$ and we fix two objects $b, c$ in $F_{a_{0}}\left(T^{\prime} \Perp_{T} T^{\prime \prime}\right)$ with $b_{T^{\prime}} \cong c_{T^{\prime}}$ resp. $b_{T^{\prime \prime}} \cong c_{T^{\prime \prime}}$ in $F_{a_{0}}\left(T^{\prime}\right)$ resp. $F_{a_{0}}\left(T^{\prime \prime}\right)$. By (2.3) (2) (ii), the canonical map

$$
\operatorname{Aut}_{T^{\prime}}^{F} a_{0}\left(b_{T^{\prime}}\right) \longrightarrow \operatorname{Aut}_{T}^{F a_{0}\left(b_{T}\right)}
$$

is surjective. From this (and $(S 1)^{\prime}$ of $[\mathbf{B i}]_{1}$ ), one obviously gets $b \cong c$ in $F_{a_{0}}\left(T^{\prime} \Perp_{T} T^{\prime \prime}\right)$, so $\left|F_{a_{0}}\right|$ is pro-representable by [Schl] Theorem 2.11. This shows that $\bar{a} \in|F|\left(S^{\prime}\right)$ is universal in $s^{\prime}$. The same argument also applies to an arbitrary point $t \in S^{\prime}$-replacing $a_{0}$ by $a_{t}$. We define the representing spaces of the appropriate functors

$$
\begin{aligned}
& G_{0}:=\operatorname{Aut}^{F}\left(a_{0}\right), \\
& G:=\operatorname{Aut}_{S^{\prime} \times S_{S} S^{\prime}}^{F}\left(p r_{1}^{*} a\right)=\operatorname{Aut}_{S^{\prime}}^{F}(a) \times{ }_{S} S^{\prime}, \\
& Z:=\operatorname{Isom}_{S^{\prime} \times S_{S} S^{\prime}}^{F}\left(\operatorname{pr}_{1}^{*} a, \operatorname{pr}_{2}^{*} a\right),
\end{aligned}
$$

where $p r_{2}: S^{\prime} \times{ }_{S} S^{\prime} \rightarrow S^{\prime}$ denotes the $i$-th canonical projection. Obviously, $G_{0}$ is the fibre of $G$ over the point $\left(s^{\prime}, s^{\prime}\right)$ and $Z$ is a formal principal homogeneous space under $G$. Moreover, the induced double arrow

$$
Z \longrightarrow S^{\prime}
$$

gives a pre-equivalence relation on $S^{\prime}$ (in the sense of [Ki]). Set-theoretically 
it is the following: Two points $s_{1}, s_{2}$ of $S^{\prime}$, lying over the same point of $S$, are equivalent, iff $a_{s_{1}}$ is isomorphic to $a_{s_{2}}$. It is our aim to construct the quotient $S^{\prime} / Z$ in the category of complex spaces. This is achieved essentially in two steps. First we consider the relative component $G^{0}$ of the unit section of $G$. By (3.10), (3.4) the quotient

$$
Z^{\prime}:=Z / G^{\circ}
$$

exists, and is a complex space over $S^{\prime} \times{ }_{S} S^{\prime}$. Moreover, $Z \rightarrow Z^{\prime}$ is smooth and surjective. We have the commuting diagram

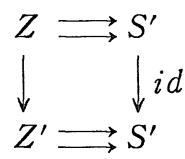

of pre-equivalence relations. If $S^{\prime} / Z^{\prime}$ exists, then $S^{\prime} / Z$ exists too and both coincide.

The next step consists by showing that the pre-equivalence relation $Z^{\prime} \rightrightarrows S^{\prime}$ is (after shrinking $S^{\prime}$ sufficiently around $s^{\prime}$ ) induced by an operation of the finite group $G_{0} / G_{0}^{0}$ on $S^{\prime}$. So $S^{\prime} / Z^{\prime}$ exists as a complex space.

(5.1) Lemma. There is a natural representation

$$
\rho: G_{0} \longrightarrow \operatorname{Aut}_{(S, s)}\left(\left(S^{\prime}, s^{\prime}\right)\right), \quad g \longmapsto \bar{\varphi}_{g}
$$

with the following properties:

(i) $\rho \mid G_{0}^{0}$ is trivial,

(ii) for each $g \in G_{0}$, there is a commutating diagram

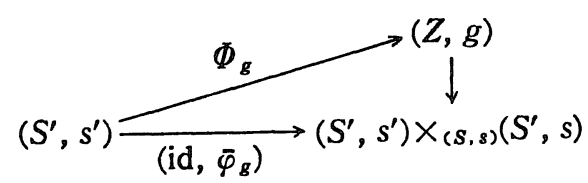

(observe that the fibre $Z_{\left(s^{\prime}, s^{\prime}\right)}$ identifies with $G_{0}$ ),

(iii) the $\Phi_{g}, g \in G$, induce the commutative diagram

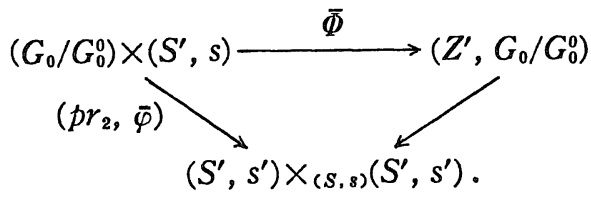

Proof. By the versality of $a$ in $s^{\prime}$, we get a commutative diagram 


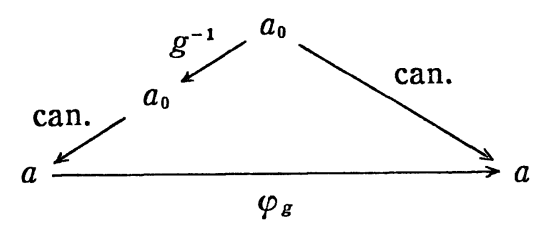

and $\varphi_{g}$ projects down to a $(S, s)$-morphism $\bar{\varphi}_{g}:\left(S^{\prime}, s\right) \rightarrow\left(S^{\prime}, s^{\prime}\right)$. Since $\bar{a}$ is even universal, $\bar{\varphi}_{g}$ is uniquely determined by $g$ and therefore the map $g \mapsto \bar{\varphi}_{g}$ is a representation. The universal property of $Z$ gives the map $\Phi_{g}$, induced by $\varphi_{g}$. Now, if $g \in G_{0}^{0}$, then $g$ can be lifted to a local section of $\operatorname{Aut}_{S^{\prime}}^{F^{\prime}}(a) \rightarrow S^{\prime}$ (see (2.3) (2) (ii)), so $\bar{\varphi}_{g}=i d$. This shows (i). Next, we take two elements $g_{1}, g_{2}$ which lie in the same connected component of $G_{0}$, i.e. $g_{2} g_{1}^{-1} \in G_{0}^{0}$. It follows that $\bar{\varphi}_{g_{1}}=\bar{\varphi}_{g_{2}}$ and $\varphi_{g_{2}}{ }^{\circ} \varphi_{g_{1}}^{-1}: a \rightarrow a$ is a morphism in $F\left(S^{\prime}\right)$. This implies (iii).-

Now, we choose $S^{\prime}$ so small that $S^{\prime}$ is invariant under the operation of $G_{0} / G_{0}^{0}$ (see (3.17)). As $\bar{\Phi}$ is an isomorphism in the distinguished fibre over $\left(s^{\prime}, s^{\prime}\right)$, and $Z^{\prime} \rightarrow S^{\prime} \times{ }_{S} S^{\prime}$ is finite (see (2.3) (2) (iii)), it is possible to assume that $\bar{\Phi}$ : $\left(G_{0} / G_{0}^{3}\right) \times S^{\prime} \rightarrow Z^{\prime}$ is a closed embedding. Next we take a point $\bar{g} \in Z_{\left(s^{\prime}, s^{\prime}\right)}^{\prime}=G_{0} / G_{0}^{0}$ and consider the map $\sigma:\left(Z^{\prime}, \bar{g}\right) \rightarrow\left(G_{0} / G_{0}^{0}\right) \times\left(S^{\prime}, s^{\prime}\right)$, given by the constant map $Z^{\prime} \rightarrow\{\bar{g}\}$ and $Z^{\prime} \rightarrow S^{\prime} \times{ }_{S} S^{\prime} \stackrel{p r_{1}}{\rightarrow} S^{\prime}$. We want to show that $\bar{\Phi}$ is a local isomorphism in $\bar{g}$ with invers $\sigma$. Let $(p, q): Z \rightarrow S^{\prime} \times{ }_{S} S^{\prime}$ denote the structure morphism. First we notice that $\bar{\varphi}_{g} \circ p=q$ on the germ $(Z, g)$. This follows immediately from the universality of $\bar{a}$ in $s^{\prime}$ and the fact that $\left(\bar{\varphi}_{g}{ }^{\circ} p\right)^{*}(a) \cong q^{*}(a)$ in $F_{a_{0}}$. Consequently the composition

$$
\chi:(Z, g) \stackrel{p}{\longrightarrow}\left(S^{\prime}, s^{\prime}\right) \stackrel{\Phi_{g}}{\longrightarrow}(Z, g)
$$

is a morphism over $S^{\prime} \times{ }_{S} S^{\prime}$. The universal property of $Z$ and (3.10) imply that the two maps $(Z, g) \stackrel{\chi}{\rightarrow}(Z, g) \stackrel{\text { can. }}{\longrightarrow}\left(Z^{\prime}, \bar{g}\right)$ and $(Z, g) \stackrel{\text { can. }}{\longrightarrow}\left(Z^{\prime}, \bar{g}\right)$ coincide, so $\bar{\Phi}$ is a local isomorphism in $\bar{g}$ with invers $\sigma$. By shrinking $S^{\prime}$ sufficiently, $\Phi$ is an isomorphism (observe that $Z^{\prime} \rightarrow S^{\prime} \times{ }_{S} S^{\prime}$ is proper). Therefore we have

$$
S^{\prime} / Z=S^{\prime} / Z^{\prime}=S^{\prime} /\left(G_{0} / G_{0}^{0}\right) \text {. }
$$

This is now the desired local piece around $s^{\prime}$ of the moduli space. The glueing procedure is done in the following way:

Let $s \in S$ be a point and put $I_{s}:=\mid F\left(\operatorname{Spec}(\kappa(s)) \mid, I:=\Perp_{s \in S} I_{s}\right.$. If we denote by $a^{i} \in F\left(S_{i}^{\prime}\right)$ the universal (local) deformation of $a_{0}^{i}$ for $i \in I$, constructed by the above method (observe $\left|a_{0}^{i}\right|=i$ ), and if we put

$$
S_{\imath \jmath}^{\prime}:=\left\{s^{\prime} \in S_{\imath}^{\prime}: \exists t \in S_{\jmath}^{\prime}: a_{s^{\prime}}^{i} \cong a_{t}^{\jmath}\right\},
$$

then $S_{\imath j}^{\prime}$ is open in $S_{\imath}^{\prime}$ and we have, via universality, isomorphisms over $S$

$$
\alpha_{\jmath 2}^{\left(t, s^{\prime}\right)}:\left(S_{\imath \jmath}^{\prime}, s^{\prime}\right) \longrightarrow\left(S_{\jmath 2}^{\prime}, t\right)
$$


for $i, j \in I_{s}$. These mappings are equivariant with respect to the pre-equivalence relations $Z_{i}$ and $Z_{j}$ on $S_{i}^{\prime}$ resp. $S_{j}^{\prime}$. Now we put $M_{i}:=S_{i}^{\prime} / Z_{i}$ and $M_{i j}:=$ $\operatorname{Im}\left(S_{i j}^{\prime} \rightarrow S_{i}^{\prime} \rightarrow M_{i}\right)$, which is open in $M_{i}$. Then the mappings above induce welldefined $S$-isomorphisms

$$
\bar{\alpha}_{j i}: M_{i j} \longrightarrow M_{j i}
$$

which fulfill the cocycle condition. Thus we obtain a global space $M$ over $S$.

It remains to show that $M$ is a coarse moduli space for $|F|$. First we have a functor $|F| \rightarrow M$, which maps an isomorphy class $b \in|F|(T)$ to the $S$ morphism $T \rightarrow M$ given by the universality of the $a^{i}$. Obviously $\left|M_{s}\right|=$ $\mid F(\operatorname{Spec}(\kappa(s)) \mid$ for each $s \in S$. Now, let $|F| \rightarrow N$ be an arbitrary $S$-morphism of functors. Considering the composition $S_{\imath}^{\prime} \stackrel{a^{i}}{\rightarrow}|F| \rightarrow N$, we can find the dotted arrow in the diagram

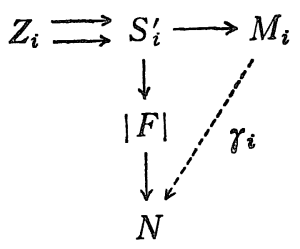

for each $i \in I$. It is immediate to see the compatibility of the $\gamma_{2}$. So we get an $S$-morphism $M \stackrel{\gamma}{\rightarrow} N$, such that

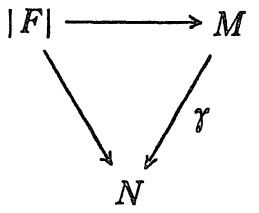

commutes. The injectivity of $\operatorname{Hom}(M, N) \rightarrow \operatorname{Hom}(|F|, N)$ is left to the reader.-

The question of separatedness of $M \rightarrow S$ :

Let $m_{1}, m_{2} \in M_{s}$ be different points for some $s \in S$ and $S_{i_{1}}^{\prime}$ resp. $S_{i_{2}}^{\prime}$ the local base spaces as above, corresponding to $m_{1}$ resp. $m_{2}$. We consider the fibre product

and further

$$
S_{i_{1}}^{\prime} \times{ }_{S} S_{i_{2}}^{\prime}
$$

$$
Z:=Z\left(\operatorname{pr}_{1}^{*}\left(a^{i_{1}}\right), \operatorname{pr}_{2}^{*}\left(a^{i_{2}}\right)\right) \text {. }
$$

Now, according to the assumption, the image $R$ of $Z$ in $S_{i_{1}}^{\prime} \times{ }_{S} S_{i_{2}}^{\prime}$ is closed. Since $m_{1}, m_{2}$ are different isomorphy classes, $R$ does not contain $\left(m_{1}, m_{2}\right)$. Therefore one has open neighbourhoods $U_{i_{1}}$ resp. $U_{i_{2}}$ of $m_{1}$ resp. $m_{2}$ in $S_{i_{1}}^{\prime}$ resp. $S_{i_{2}}^{\prime}$ with $R \cap\left(U_{i_{1}} \times{ }_{S} U_{i_{2}}\right)=\varnothing$. The image of $U_{i_{1}} \times{ }_{S} U_{i_{2}}$ in $M \times{ }_{S} M$ is open and does not meet the diagonal $\Delta_{M / S}$. This shows that $\left(M \times_{S} M\right) \backslash \Delta_{M / S}$ is open.- 


\section{§6. An Application to Moduli of Simple Coherent Sheaves}

In this section we are going to show that on a fixed compact complex space $X$, there exists a coarse moduli space of simple coherent sheaves and especially of simple holomorphic vector bundles. Our result contains the one of A. Norton (see [No] Theorem 4), where $X$ is assumed to be smooth. We begin with some preparations.

(6.1) Proposition. Let $f: X \rightarrow S$ be a separated map of complex spaces and $\mathscr{I}, \mathcal{G}$ be coherent sheaves on $X$ which are flat and with proper support over $S$.

Then the complex space $\operatorname{Isom}_{X}(\mathscr{I}, \mathcal{G})$ is, locally in $S$, compactifiable over $S$.

Proof. We consider the complex spaces over $S$ (belonging to the appropriate functors):

$$
\begin{aligned}
& Z:=\operatorname{Isom}_{X}(\mathscr{F}, \mathcal{G}), \\
& V_{1}:=\operatorname{Hom}_{X}(\mathscr{F}, \mathcal{G}), \quad V_{2}:=\operatorname{Hom}_{X}(\mathcal{G}, \mathscr{I}), \\
& W_{1}:=\operatorname{End}_{X}(\mathscr{F}), \quad W_{2}:=\operatorname{End}_{X}(\mathscr{G}) .
\end{aligned}
$$

Then the $V_{i}$ and $W_{i}$ are linear fibre spaces over $S$. Let $\alpha: V_{1} \times{ }_{S} V_{2} \rightarrow W_{1} \times{ }_{S} W_{2}$ be the $\operatorname{map}(\varphi, \phi) \mapsto(\psi \varphi, \varphi \psi)$. If we set $\tilde{Z}:=\alpha^{-1}((i d, i d))$, then $Z=\operatorname{Im}\left(\tilde{Z} \subset V_{1} \times_{S} V_{2}\right.$ $\left.\stackrel{p r_{1}}{\rightarrow} V_{1}\right)$. Since $V_{\imath}, W_{\imath}, \tilde{Z}$ and $\alpha$ are relatively algebraic, we get by Chevalley's theorem that $Z \subset V_{1}$ is constructible. Denote by $\bar{V}_{1} \rightarrow S$ the natural projective compactification of $V_{1} \rightarrow S$. Then also $Z \subset \bar{V}_{1}$ is constructible and open in the complex topology, so $Z$ is Zariski-open in $\bar{V}_{1}$.-

(6.2) Definition. Let $f: X \rightarrow S$ be a separated holomorphic map of complex spaces and $\mathscr{I}$ a coherent $\mathcal{O}_{X}$-module, flat and proper over $S$. Then $\mathscr{F}$ is called simple (over $S$ ), iff the canonical homomorphism

$$
\begin{aligned}
& S \times \boldsymbol{C} \longrightarrow \operatorname{End}_{X}(\mathscr{I}) \\
& (s, t) \longmapsto t \cdot i d_{\mathscr{F}(s)}
\end{aligned}
$$

is an isomorphism of linear fibre spaces over $S . *)$

(6.3) Remark. (1) The trivial sheaf is not simple.

(2) $\mathbb{F}$ is simple over $S$, iff each $\mathbb{F}(s), s \in S$, is simple.

(3) Simpleness is an open condition in $S$.

Let $X \rightarrow S$ be a separated map of complex spaces. We denote by

$$
\mathrm{Coh}_{\mathrm{s}}(X / S)
$$

the groupoid over $(\mathrm{An} / \mathrm{S})$ of simple coherent sheaves. An object of this category

*) It has been pointed out to us by M. Maruyama that this definition is adapted to the case where $f$ is reduced with connected fibres 
is given by a complex parameter space $T / S$ together with a simple (over $T$ ) coherent $\mathcal{O}_{X_{T}}$-module.

(6.4) Theorem. The functor

$$
\begin{aligned}
F:(\mathrm{An} / S) & \longrightarrow(\text { sets }) \\
T & \longmapsto\left|\operatorname{Coh}_{s}(X / S)(T)\right|
\end{aligned}
$$

of isomorphy classes of simple coherent sheaves on X/S has a coarse moduli space. Moreover, the sheafified functor $F^{\#}$ of $F$ is representable.

Proof. We want to apply theorem (2.2) to the groupoid $\mathrm{Coh}_{s}(X / S) \rightarrow(\mathrm{An} / S)$. Condition (2.2) (1) is satisfied according to (6.3) and the theorem of Siu/Trautmann $[\mathbf{S}-\mathbf{T}]$. Observe that also openness of versality holds (see $[\mathbf{B i}]_{1} \S 8$ ). Using well-known results and (6.2), conditions (2)(i), (2) (ii) are fulfilled too. It remains to verify (2) (iii). For this, let $\mathscr{I}$ and $\mathcal{G}$ be simple sheaves on $X_{T}$ and put $Z:=\operatorname{Isom}_{X_{T}}(\mathscr{F}, \mathcal{G}), G:=\operatorname{Aut}_{X_{T}}(\mathscr{I})$. By (3.16), it is sufficient to show that $Z \rightarrow T$ is of locally constant connectedness type. Proposition (6.1) and (3.15) gives condition (3.14) (1), so we have to verify (3.14) (2). We fix a point $t \in T$ and assume $Z_{t} \neq \varnothing$. With the notations of (3.14), let $Z_{U_{0}}^{\prime}$ be a connected component of $Z_{U_{0}}$ and $\varphi_{\nu} \in Z_{U_{\nu}}^{\prime}:=\left(Z_{U_{0}}^{\prime}\right)_{U_{\nu}}$ a point for each $\nu$. We shall show: Modulo passing to a subsequence, there are positive real numbers $c_{\nu} \in \mathbb{R}_{>0}$, such that $c_{\nu} \cdot \varphi_{\nu} \in Z_{U_{\nu}}^{\prime}$ and $\left(c_{\nu} \varphi_{\nu}\right)_{\nu}$ converges to a point $\varphi \in Z_{t}$. On the linear fibre space $H:=\operatorname{Hom}_{X_{T}}(\mathscr{F}, \mathcal{G})$ over $T$, we fix a differentiable $T$-morphism

$$
\varepsilon: H \longrightarrow T \times \boldsymbol{R}_{\geqq 0}
$$

with the following properties:

(1) $\varepsilon^{-1}(T \times\{0\})=$ zero section of $H$,

(2) the sets $\left\{p r_{2} \varepsilon \leqq a\right\}$ are proper over $T$ for each $a \in \mathbb{R}_{\equiv 0}$,

(3) $p r_{2} \varepsilon(c h)=|c| p r_{2} \varepsilon(h)$, for $h \in H$ and $c \in \mathbb{R}$.

This is a kind of Finsler-metric on $H$. We set

$$
r_{\nu}:=\left(p r_{2} \circ \varepsilon\right)\left(\varphi_{\nu}\right) \text {, }
$$

then $r_{\nu}>0$, since we have $\varphi_{\nu} \neq 0$ for all $\nu$. Putting $c_{\nu}:=\left(1 / r_{\nu}\right)$, we get $\left(p r_{2} \circ \varepsilon\right)$ $\left(c_{\nu} \varphi_{\nu}\right)=1$. By (2) (and modulo passing to a subsequence), the sequence $\left(c_{\nu} \varphi_{\nu}\right)_{\nu}$ converges to a $\varphi \in H_{t}$. Now, since all $Z_{U_{\nu}}^{\prime}$ are invariant under multiplication by numbers $c \in C^{*}$, we have $c_{\nu} \varphi_{\nu} \in Z_{U_{\nu}}^{\prime}$. Obviously, $\varphi \neq 0$, since $\left(p r_{2} \circ \varepsilon\right)(\varphi)=1$. Our assumption was $Z_{t} \neq \varnothing$, so there is at least one isomorphism $\mathscr{I}(t) \stackrel{\phi}{\rightarrow} \mathscr{G}(t)$. We conclude that $\varphi^{\circ} \psi^{-1}$ is a non-trivial endomorphism of $\mathcal{G}(t)$, and, since $\mathcal{G}(t)$ is simple, this implies that $\varphi$ itself is an isomorphism. Thus the theorem is established.-

As a consequence, we have 
(6.5) Corollary. There is a coarse moduli space of simple holomorphic bundles on a fixed compact complex space.

In general these moduli spaces need not to be separated, but there is the following criterion for separateness (compare also [No] Prop. 2 on page 370).

(6.6) Proposition. Let $\mathscr{I}_{0}, \mathcal{G}_{0}$ be non-isomorphic simple coherent sheaves on the complex space $X$. If the associated points to $\mathscr{I}_{0}$ and $\mathcal{G}_{0}$ are non-separated in the moduli space of simple sheaves on $X$, then there are non-trivial homomorphisms $\varphi: \mathscr{I}_{0} \rightarrow \mathcal{G}_{0}$ and $\phi: \mathfrak{G}_{0} \rightarrow \mathscr{I}_{0}$ with $\varphi \psi=0, \phi \varphi=0$.

Proof. Let $\mathscr{I}$ resp. $\mathcal{G}$ denote the local semi-universal deformation of $\mathscr{T}_{0}$ resp. $\mathcal{G}_{0}$ over the space germ $(T, 0)$ resp. $\left(T^{\prime}, 0\right)$. We set

and

$$
H:=\operatorname{Hom}_{T>T^{*} \times X}\left(\mathscr{F}_{T \times T^{\prime}}, \mathcal{G}_{T \times T^{\prime}}\right),
$$

$$
Z:=\operatorname{Isom}_{T \times T^{\prime} \times X}\left(\mathscr{F}_{T \times T^{\prime}}, \mathcal{G}_{T \times T^{*}}\right) .
$$

Furthermore, we fix a differentiable function $\varepsilon: H \rightarrow T \times T^{\prime} \times \boldsymbol{R}_{\geqq 0}$ as in the proof of (6.4). By assumption, there is a sequence of points $\varphi_{\nu} \in Z$ with base points $\left(t_{\nu}, t_{\nu}^{\prime}\right) \in T \times T^{\prime}$, such that $\left(t_{\nu}, t_{\nu}^{\prime}\right) \underset{\nu \rightarrow \infty}{\longrightarrow}(0,0)$. We may assume $\left(p r_{3} \varepsilon\right)\left(\varphi_{\nu}\right)=c \neq 0$ for all $\nu$ and $\varphi_{\nu} \underset{\nu \rightarrow \infty}{\longrightarrow} \varphi \in H_{(0,0)}$, where $\varphi$ is non-trivial (compare the proof of $(\mathbf{6 . 4})$ ). Reversing the roles of $\mathscr{I}$ and $\mathcal{G}$, we get a non-trivial $\phi: \mathscr{G}_{0} \rightarrow \mathscr{I}_{0}$. Now $\phi \varphi=0$, $\varphi \psi=0$, otherwise $\varphi$ and $\phi$ would be isomorphisms, which is absurd, since $Z_{(0,0)}$ $=\varnothing$.-

Let $F:(\mathrm{An} / S) \rightarrow$ (sets) be the functor in (6.4). We can give the following alternative description of $F^{\#}$ :

Let $T$ be a space in $(\mathrm{An} / S)$ and $\mathscr{I}, \mathcal{G}$ be simple sheaves on $X_{T} / T$. We call $\mathscr{I}$ and $\mathcal{G}$ equivalent, iff there is a line bundle $\mathcal{L}$ on $T$ and an isomorphism $\mathscr{F} \cong f_{T}^{*}(\mathcal{L}) \otimes \mathcal{G}$, where $f: X \rightarrow S$ is the structure morphism. The set of such equivalence classes is denoted by $\bar{F}(T)$. Then we have

(6.7) Remark. The canonical morphism $F \rightarrow \bar{F}$ induces an isomorphism

$$
F^{\#} \longrightarrow \bar{F}^{+},
$$

where "+" means the associated Čech-construction of a pre-sheaf.

Proof. Let $T=\cup_{i} T_{i}$ be an open covering of $T$. It is easy to see that $\bar{F}(T) \rightarrow \prod_{i \in I} \bar{F}\left(T_{i}\right)$ is injective. So $\bar{F}^{+}$is already a sheaf and therefore we get a morphism $F^{\#} \rightarrow \bar{F}^{+}$, which is obviously an isomorphism.-

The operation of the Picard-functor. 
Let $f: X \rightarrow S$ be a separated holomorphic map, $F:(\mathrm{An} / S) \rightarrow($ sets $)$ the functor of isomorphy classes of simple sheaves on $X / S$ and $P i c_{X / S}:(\mathrm{An} / S) \rightarrow$ (groups) the relative Picard-functor given by $T \rightarrow \Gamma\left(T, R^{1}\left(f_{T}\right)_{*}\left(\mathcal{O}_{X_{T}}^{*}\right)\right)$. Then we have an operation

$$
P i c_{X / S} \times F^{\#} \longrightarrow F^{\#}
$$

induced by tensorization with line bundles. If $P i c_{X / S}$ is representable (this is the case when $f$ is proper, flat and cohomologically flat in dimension 0 , see $[\mathbf{B i}]_{2}$ ) by the Liegroup $G \rightarrow S$ (not necessarily separated over $S$ ), we get an operation

$$
G \times{ }_{S} M \longrightarrow M
$$

of $G$ on the moduli space $M$ of simple sheaves on $X / S$.

Now, denote by $\operatorname{Proj}(X / S)$ the groupoid over $(\mathrm{An} / S)$, whose objects over $T$ are projective fibre spaces $P \rightarrow X_{T}$, such that $P$ is, locally in $T$, induced by a simple sheaf on $X_{T} / T$. The morphisms in this category are morphisms of projective fibre spaces, locally in $X_{T}$ given by isomorphisms of coherent modules. Let

$$
F^{\prime}:(\mathrm{An} / S) \longrightarrow(\text { sets })
$$

be the functor of isomorphy classes of such projective bundles.

(6.8) Proposition. The functor morphism $F \rightarrow F^{\prime}$, given by $\mathscr{F} \rightarrow \boldsymbol{P}(\mathscr{I})$ induces an epimorphism

$$
\left(F^{\#} / P i c_{X / S}\right)^{\#} \longrightarrow F^{\prime \#} \text { 。 }
$$

This is an isomorphism, if we replace sheaves by bundles.

The proof is an immediate consequence of standard properties of projective fibre bundles.

(6.9) Corollary. Assume that the quotient $M / G$ exist as a complex space over $S$. Then $M / G$ is a coarse moduli space for projective bundles associated to simple bundles on $X / S$.

Proof. By using (6.8), it is sufficient to show that $\left(h_{M / S} / h_{G / S}\right)^{\#}$ has $M / G$ as a coarse moduli space. But this is clearly satisfied. Observe that a coarse moduli space for $F^{\prime \#}$ is also one for $F^{\prime}$ and vice versa.-

\section{References}

$[\mathrm{Bi}]_{1}$ Bingener, J., Offenheit der Versalität in der analytischen Geometrie, Math. Z., 173 (1980), 241-281.

$[\mathrm{Bi}]_{2}$ Bingener, J., Darstellbarkeitskriterien für analytische Funktoren, Ann scient. Éc. Norm. Sup. $4^{e}$ série, t. 13, 1980, p. 317 à 347. 
[Bi-Fl] Bingener, J., Flenner, H., Constructible and quasiconstructible sheaves on analytic spaces, Abh. Math. Sem. Univ. Hamburg, 54 (1984), 119-139.

[Go-MP] Goresky, M., MacPherson, R., Intersection homology II, Invent. math., 72 (1983), 77-129.

[Ki] Kiehl, R., Äquivalenzrelationen in analytischen Räumen, Math. Z., 105 (1968), $1-20$.

[Ko] Kosarew, S., Grothendieck's existence theorem in analytic geometry and related results, to appear.

[Lü-Ok] Lübke, M., Okonek, C., Moduli spaces of simple bundles and Hermitian-Einstein Connections, Math. Ann. 276 (1987), 663-674.

[No] Norton, A., Analytic Moduli of Complex Vector Bundles, Indiana Univ. Math. J., 28 (1979), 365-387.

[Schl] Schlessinger, M., Functors of Artin rings, Trans. AMS, 130 (1968), 208-222.

$[\mathrm{Schu}]_{1}$ Schumacher, G., Construction of the Coarse Moduli Space of Compact Polarized Kähler Manifolds with $c_{1}=0$, Math. Ann., 264 (1983), 81-90.

[Schu $]_{2}$ Schumacher, G., Moduli of Polarized Kähler Manifolds, Math. Ann., 269 (1984), 137-144.

[S-T] Siu, Y.T., Trautmann, G., Deformations of coherent analytic sheaves with compact supports, Memoirs Am. Math. Soc., 238 (1981).

[S-V] Schuster, H.W., Vogt, A., The moduli of quotients of a compact complex space, J. reine u. ang. Math., 364 (1986), 51-59.

[SGA] Bertin, J.E., Géneralités sur les préschemas en groupes, Exp. VI $I_{B}$ in: Sém. Géom. Alg. SGA3, Lect. Notes in Math., 151, Springer V., Berlin-HeidelbergNew York (1970). 
